\title{
Improving a Single Down-Sampled Image Using Probability-Filtering-Based Interpolation and Improved Poisson Maximum A Posteriori Super-Resolution
}

\author{
Min-Cheng Pan \\ Department of Computer Science and Information Engineering, Tung-Nan Institute of Technology, \\ Shenkeng, Taipei County 222, Taiwan
}

Received 29 November 2004; Revised 25 March 2005; Accepted 4 May 2005

\begin{abstract}
We present a novel hybrid scheme called "hyper-resolution" that integrates image probability-filtering-based interpolation and improved Poisson maximum a posteriori (MAP) super-resolution to respectively enhance high spatial and spatial-frequency resolutions of a single down-sampled image. A new approach to interpolation is proposed for simultaneous image interpolation and smoothing by exploiting the probability filter coupled with a pyramidal decomposition and the Poisson MAP super-resolution is improved with the techniques of edge maps and pseudo-blurring. Simulation results demonstrate that this hyper-resolution scheme substantially improves the quality of a single gray-level, color, or noisy image, respectively.
\end{abstract}

Copyright (C) 2006 Min-Cheng Pan. This is an open access article distributed under the Creative Commons Attribution License, which permits unrestricted use, distribution, and reproduction in any medium, provided the original work is properly cited.

\section{INTRODUCTION}

Basically, the quality of an image can be evaluated on its spatial and spatial-frequency resolutions. Image interpolation and super-resolution are perhaps the way to respectively produce high spatial and spatial-frequency resolutions of images especially for a single down-sampled image. For convenience, the term "hyper-resolution" used here represents the approach to enhancing the spatial and the spatial-frequency resolutions of an image. Some algorithms have been proposed in the literature [1-6], where a high resolution image is reconstructed by combining multiple low resolution images and incorporating with some constraints for the specific purpose. This paper will consider hyper-resolution for a single gray-level or color image without any constraint. Furthermore, hyper-resolution of a noisy image is also performed; generally, the procedure for processing noisy images is shown in Figure 1(a), that is, noise removal, interpolation, and then super-resolution; whereas the proposed scheme is dealing with interpolation and noise removal simultaneously, as shown in Figure 1(b).

As known, the process of decimation or down-sampling is an effective way aid often used to reduce image sizes; thus, reducing the amount of information transmitted through the communication channels and the local storage requirements, while trying to preserve as much as possible the image quality. Conversely, the reverse procedure of this, referred to as interpolation or up-sampling, is useful in restoring the original high resolution image from its decimated version or for resizing or zooming a digital image. Decimation and interpolation are used for several purposes in many practical applications, such as progressive image transmission systems, multimedia applications, and so forth. These problems are further aggravated in the case of color images which usually require larger storage capacity and processing time.

A number of conventional interpolation techniques [79] have been proposed to increase the spatial resolution of an image. Undoubtedly, these techniques degrade quality of the magnified image. Those degradations become worse as the magnification ratio increases and there also exists tradeoff between reducing blocking artifacts and excessive smoothness [10]. Often, image interpolation is performed by pixel replication in a small neighborhood of each existing pixel. This is equivalent to a first-order linear filter. Obviously, such a scheme is very simple, but can hardly provide a satisfactory solution. The performance can be slightly improved by employing higher-order linear filters $[11,12]$. However, it seems that there do not exist good criteria for the design of optimal linear interpolators. As known, linear interpolation is generally preferred not for the performance but for computational 


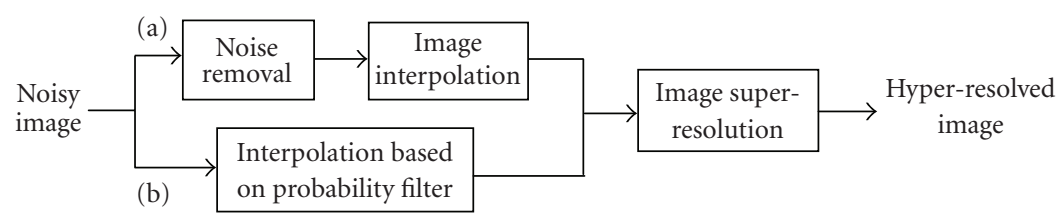

Figure 1: The block diagram of hyper-resolution for a noisy image. (a) Conventional approach and (b) proposed approach.

simplicity whereas adaptive methods [13-15] aim to avoid these problems by analyzing the local structure of the source image and imposing more accurate models. However, few of them concern the problem of dealing with noisy images.

Following the procedure of interpolation, image superresolution is perhaps the only way to reduce blurring effects resulting from smoothing or interpolation. In recent years it has become clear that there are credible methods [16-21] for the reconstruction of spatial frequencies of the object that are greater than the diffraction-limit spatial frequency in the image. Specifically, processes that attempt to extend the spatialfrequency spectra beyond the image passband are usually referred to as super-resolution algorithms.

This paper first addresses an approach to simultaneous image interpolation and smoothing by exploiting the probability filter [22] coupled with a pyramidal decomposition, thereby extending the conventional applications of the probability filter originally designed for noise removal. Then, the improved Poisson maximum a posteriori (MAP) super-resolution [23] is performed to reconstruct the high spatial-frequency spectrum of the interpolated image. Thus, the hybrid scheme shown in Figure 1(b) is proposed for enhancing the spatial and the spatial-frequency resolutions of down-sampled images. To illustrate the performance of the proposed scheme, this research has studied the resolution enhancement on gray-level, color, and noisy images, respectively, and comparisons will be made among the superresolution coupled with different interpolators.

Following the above introduction, this paper is organized as follows: Section 2 briefly reviews the B-spline interpolation including bilinear and cubic spline interpolators incorporated in the hyper-resolution scheme for comparison. Section 3 details the hyper-resolution scheme proposed here for down-sampled images. Experimental results and evaluations are shown in Section 4 and finally some conclusions are drawn in Section 5.

\section{B-SPLINE INTERPOLATION}

Recently, image-resizing algorithms using high-order Bsplines have been published $[24,25]$. The standard interpolation procedure is to fit the original data with a continuous model and then resample function on the grid appropriate to the scaling desired [26]. The B-spline interpolation can be modeled as follows:

$$
s(x)=\sum_{k \in Z} c(k) \beta^{n}(x-k)
$$

where $c(k)$ is the coefficient, $\beta^{n}(x)$ is the B-spline of degree $n$. The B-splines are symmetrical, bell-shaped functions constructed from the $(n+l)$-fold convolution of a rectangular pulse $\beta^{0}(x)$, shown as below:

$$
\begin{gathered}
\beta^{0}(x)= \begin{cases}1, & |x|<\frac{1}{2} \\
\frac{1}{2}, & |x|=\frac{1}{2} \\
0, & \text { otherwise, }\end{cases} \\
\beta^{n}(x)=\beta^{n-1} \otimes \beta^{0}=\beta^{0} \otimes \beta^{0} \otimes \beta^{0} \otimes \cdots \otimes \beta^{0}(n+1) \text { times. }
\end{gathered}
$$

Equation (2) shows that all B-splines are positive and have an integral that is equal to one. Among these splines, the cubic spline has been popular in applications due to their minimum curvature property. The central spline of degree 3 is given by

$$
\beta^{3}(x)= \begin{cases}\frac{2}{3}-|x|^{2}+\frac{|x|^{3}}{2}, & 0 \leq|x| \leq 1 \\ \frac{(2-|x|)^{3}}{6}, & 1 \leq|x| \leq 2 \\ 0, & |x| \leq 2\end{cases}
$$

Usually, lower-order interpolation algorithms are simplest and fastest, but they tend to produce artifacts. The Bspline of degree 0 , the nearest neighborhood interpolation, selects one of the upper, lower, left, and right four pixels. In case of the B-spline of degree 1, the bilinear interpolation, the weighted average value of the upper, lower, left, and right four pixels is assigned. The B-spline of degree 3, the cubic spline interpolation, produces better outcomes but requires much more computation. Generally, among these interpolation algorithms, the bilinear interpolation has been widely used in digital still cameras. However, there is a limitation for capturing high quality images since the bilinear interpolation method tends to smooth images and result in noticeable color artifacts along edges [27].

\section{NOVEL HYPER-RESOLUTION SCHEME}

A hyper-resolution scheme proposed here is a hybrid approach, including a novel probability-filtering-based interpolator and the improved Poisson MAP super-resolution algorithm for enhancing the spatial resolution with noise removal and the spatial-frequency resolution, respectively. 


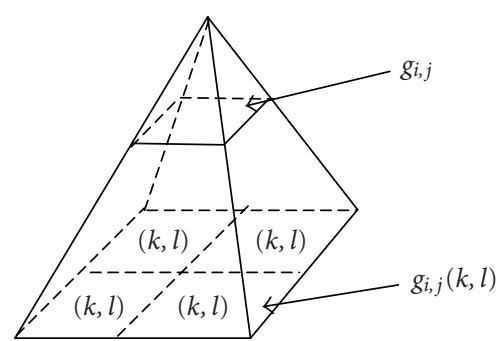

(a)

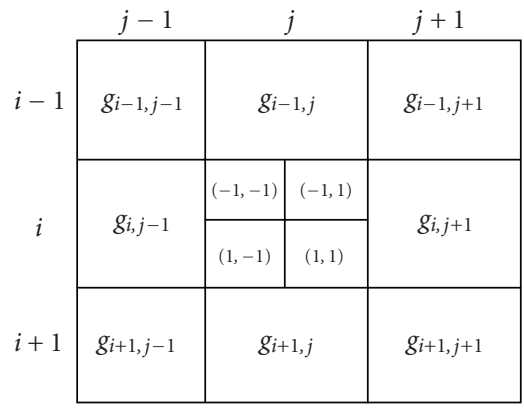

(b)

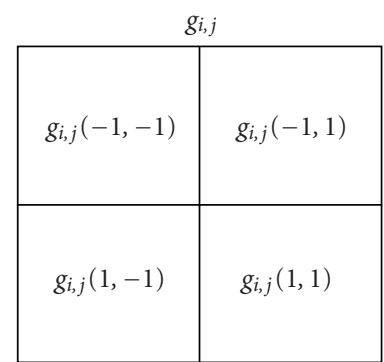

(c)

FIGURE 2: The pyramidal decomposition for interpolation. (a) Diagram of pyramidal decomposition, (b) the notation and relation of interpolated and original pixels, and (c) $g_{i, j}$ decomposed into $g_{i, j}(-1,-1), g_{i, j}(-1,1), g_{i, j}(1,-1)$, and $g_{i, j}(1,1)$.

\subsection{Interpolation based on the probability filter}

Originally, a probability filter [22] is designed for image smoothing or integration with variable weights which are interpreted as probabilities of respective pixel values in a local neighborhood of the current estimated pixel. The algorithm of this filter is explained in more detail as follows. $f^{*}(i, j)$, the restored pixel at $(i, j)$, is defined as

$$
f^{*}(i, j)=\sum_{k=-n}^{n} \sum_{l=-n}^{n} p(i+k, j+l) g(i+k, j+l),
$$

where $p(i+k, j+l)$, the probability function of a $(2 n+1)$ by $(2 n+1)$ mask, is given as below:

$$
\begin{gathered}
\begin{array}{c}
p(i+k, j+l) \\
=\frac{\exp \left(-(g(i+k, j+l)-\bar{g}(i, j))^{2} / 2 T_{1}^{2}\right)}{\operatorname{Norm} 1}, \\
k, l=-n, \ldots,-1,0,1, \ldots, n,
\end{array} \\
\text { Norm } 1=\sum_{k=-n}^{n} \sum_{l=-n}^{n} \exp \left(-\frac{(g(i+k, j+l)-\bar{g}(i, j))^{2}}{2 T_{1}^{2}}\right), \\
T_{1}=(2 n+1)^{-2} \sum_{k=-n}^{n} \sum_{l=-n}^{n}|g(i+k, j+l)-\bar{g}(i, j)|
\end{gathered}
$$

where $\bar{g}(i, j)$ and $g(i+k, j+l)$ are the average of all pixels in the mask centered at $(i, j)$ and the gray level of the pixel at $g(i+k, j+l)$, respectively.

The difference function, $(g(i+k, j+l)-\bar{g}(i, j))$, is used in (5) rather than the gray level difference between the neighbor pixel and the center pixel, thereby leading to the proposed filter being more suitable for removing impulse noise because it has a rather small probability even as the center pixel is impulse noise. If $T_{1}$ is infinite, the probability filter is identical to the mean filter. From the discussion above, the probability filter employs over a range of behavior between mean-like and median-like filtering. Furthermore, no a priori knowledge is necessary for $T_{1}$; even in the case of the image corrupted by unknown noise, $T_{1}$ can be straightforwardly determined as (6).

For the purpose of image interpolation with noise removal, this probability filter is modified as follows. According to the pyramidal decomposition shown in Figure 2, the new interpolated pixel, $g_{i, j}(k, l)$, yielded from the original pixel, $g_{i, j}$, can be defined as below:

$$
\begin{aligned}
& g_{i, j}(k, l) \\
& \quad=\frac{\left(p_{i, j} g_{i, j}+p_{i+k, j} g_{i+k, j}+p_{i, j+l} g_{i, j+l}+p_{i+k, j+l} g_{i+k, j+l}\right)}{\operatorname{Norm} 2},
\end{aligned}
$$

where

$$
\begin{gathered}
p_{i, j}=w \cdot \exp \left(-\frac{\left(g_{i, j}-\bar{g}\right)^{2}}{2 T_{2}^{2}}\right), \\
p_{i+k, j}=\exp \left(-\frac{\left(g_{i+k, j}-\bar{g}\right)^{2}}{2 T_{2}^{2}}\right), \\
p_{i, j+l}=\exp \left(-\frac{\left(g_{i, j+l}-\bar{g}\right)^{2}}{2 T_{2}^{2}}\right) \\
p_{i+k, j+l}=\exp \left(-\frac{\left(g_{i+k, j+l}-\bar{g}\right)^{2}}{2 T_{2}^{2}}\right), \\
\operatorname{Norm} 2=p_{i, j}+p_{i+k, j}+p_{i, j+l}+p_{i+k, j+l}, \\
\bar{g}=\frac{\left(g_{i, j}+g_{i+k, j}+g_{i, j+l}+g_{i+k, j+l}\right)}{4}, \\
T_{2}=\frac{\left(\left|g_{i, j}-\bar{g}\right|+\left|g_{i+k, j}-\bar{g}\right|+\left|g_{i, j+l}-\bar{g}\right|+\left|g_{i+k, j+l}-\bar{g}\right|\right)}{4},
\end{gathered}
$$

and $w$, a weight number, is chosen to be 1.5 here and $(k, l)=$ $(-1,-1),(-1,1),(1,-1),(1,1)$ shown as Figure 2.

As in the previous discussion, the modified definition, (7) (8), employs the characteristics of interpolating an image with noise removal. 


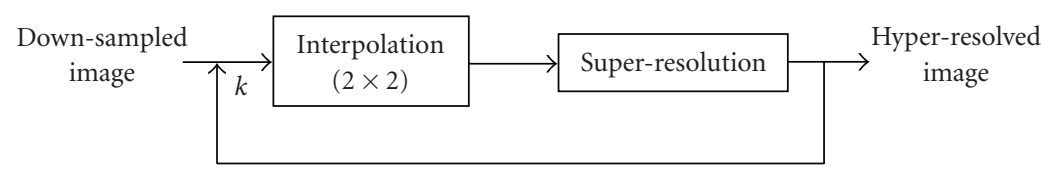

Figure 3: The mechanism of a hyper-resolution loop.

\subsection{Improved Poisson MAP super-resolution algorithm}

For brief description here, the Poisson MAP [16, 17] is given and defined as follows:

$$
\hat{f}_{n+1}=\hat{f}_{n} \exp \left[\left(\frac{g}{\hat{f}_{n} \otimes h}-1\right) * h\right]=\hat{f}_{n} C,
$$

where $\otimes$ represents a convolution, $*$ a correlation, and $h$ the point spread function (PSF); $g$ is the interpolated image and $\widehat{f}$ the final hyper-resolved image; $\hat{f}_{0}$ is defined as $g$; subscript $n$ is the iteration number; and

$$
C=\exp \left[\left(\frac{g}{\hat{f}_{n} \otimes h}-1\right) * h\right]
$$

Here, $C$ can be regarded as the correction term during the iterative restoration process. In terms of the operation of the Poisson MAP, it is an iterative algorithm where a successive estimate of the restored image is obtained by multiplication of the current estimate by a quantity close to one. The quantity close to one is a function of the interpolated image divided by a convolution of the current estimate with the PSF. Following that, the Poisson MAP can be improved by itself by operating upon the edge map with a reblurring technique; that is, the $g$ and $\hat{f}_{n}$ of the Poisson MAP are replaced by the corresponding gradients of the $g \otimes h$ and $\hat{f}_{n}$ along with the integrated PSF $(h \otimes h)$. Mathematically, it is shown that

$$
\left(\hat{f}_{n+1}\right)^{\prime}=\left(\hat{f}_{n}\right)^{\prime} \exp \left[\left(\frac{(g \otimes h)^{\prime}}{\left(\hat{f}_{n}\right)^{\prime} \otimes(h \otimes h)}-1\right) *(h \otimes h)\right] .
$$

Thus, the final hyper-resolved image $\hat{f}$ can be obtained by integrating $\left(\hat{f}_{n+1}\right)^{\prime}$. The whole process of this improved Poisson MAP includes reblurring, differentiation, restoration, integration, and then correction for a DC offset. More details about this algorithm can be found in the author's previous work [23].

\subsection{Analysis of the hyper-resolution scheme}

As discussed in the previous subsections, the filter originally designed for image smoothing can be extended for image interpolation. With the comparison between B-spline and probability-filtering-based interpolation, it can be found that bilinear or cubic spline interpolation performs on an image as weighted mean filtering while the probability-filteringbased interpolation acts as an adaptive filtering useful for preserving image features in addition to image interpolation; that is because the former creates interpolated pixels simply by averaging their own neighborhood pixels and the latter analyzes the characteristics of surrounding pixels prior to interpolating pixels. This analysis of image features is based on the probability of gray-level values and thus features such as edges are of higher probabilities than flat regions around features; furthermore, noisy images containing impulse noise can be removed completely since a single high or low graylevel pixel has a low probability. Therefore, image superresolution will perform on the interpolated image using the probability-filtering method more effectively than using the bilinear or cubic spline method.

\section{EXPERIMENTAL RESULTS AND EVALUATIONS}

To assess the performance of our proposed hyper-resolution scheme, the resolution enhancement of gray-level, color, and noisy images is considered. In illustrated examples, a graylevel image of clown with the size of $200 \times 320$ pixels and a color image of lotus with the size of $100 \times 100$ pixels were rectangular decimated with a factor of $1 / 2^{2 k}$. Therefore, the interpolation was done with a factor of $2^{2}$ for each cycle; then, the super-resolution procedure of the proposed hyper-resolution scheme, shown in Figure 3, adopted 25 iterations and the PSF with a standard deviation of 1 pixel because of the pyramidal decomposition used here, conversely, four neighboring pixels in the interpolated image mainly contributing to one pixel in its own decimated image. Thus, the proposed hyperresolution scheme can magnify the size of an image by any factor that is a power of two along each dimension where this resizing scheme consists of two steps: the first step is to interpolate the down-sampled image using bilinear, cubic spline, or the novel proposed probability-filtering-based interpolation and the second step is to super-resolve the interpolated image. The implementations of bilinear and cubic spline interpolation are taken from Matlab 5.3. In the following, it can be seen that hyper-resolved images derived from the conventional interpolators still have blocky, jagged lines, and are blurred whereas the proposed method appears to suppress these artifacts, preserve the edges, and retain the image details better.

In experiments, the peak signal-to-noise ratio (PSNR) was used to compare the image qualities quantitatively and is defined as follows:

$\operatorname{PSNR}(\mathrm{dB})$

$$
=10 \log _{10}\left[\frac{255^{2}}{(1 / M N) \sum_{i=0}^{M-1} \sum_{j=0}^{N-1}(O(i, j)-H(i, j))^{2}}\right],
$$




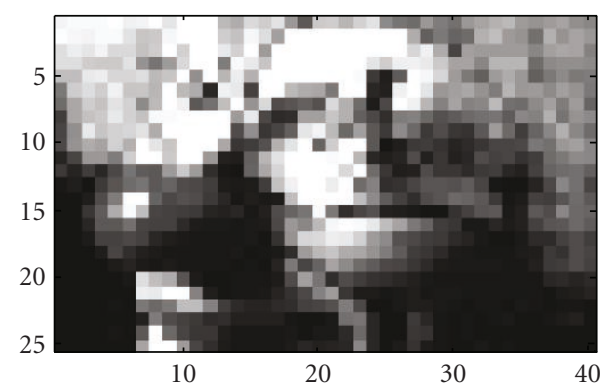

(a)

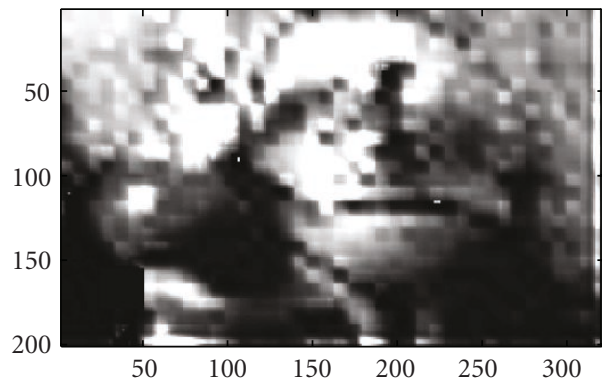

(c)

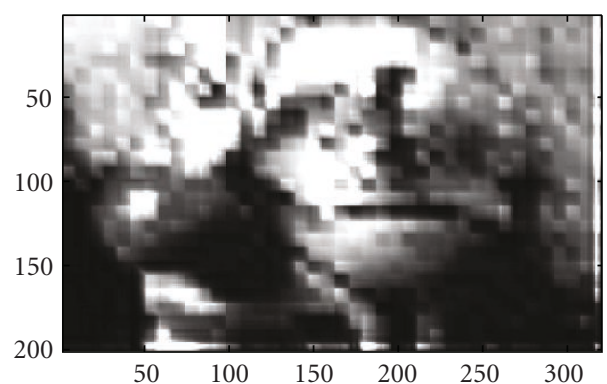

(b)

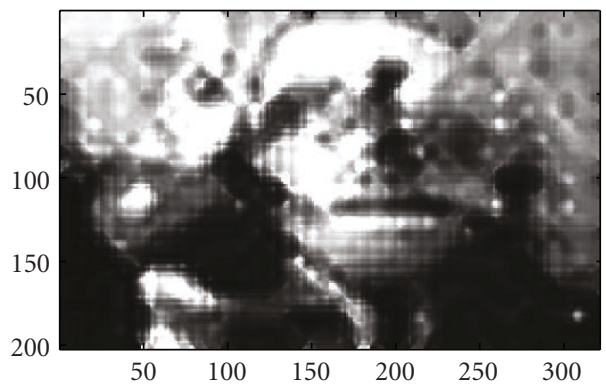

(d)

FIGURE 4: Demonstration of hyper-resolution for a single down-sampled gray-level image. (a) Down-sampled image, (b) hyper-resolved image incorporated with bilinear interpolation, (c) hyper-resolved image incorporated with cubic spline interpolation, and (d) hyper-resolved image incorporated with probability-filtering-based interpolation.

where $[M, N]$ is the image size, 255 is the maximum value that a pixel can have for an 8 -bit image. $O(i, j)$ and $H(i, j)$ are an original image and a hyper-resolved image, respectively.

\subsection{Resolution enhancement of gray-level images}

To compare the hyper-resolution effect, the down-sampled image was hyper-resolved repeatedly for resizing it to its initial dimension. As an example, consider Figure 4 where a single down-sampled image, Figure 4(a), was hyper-resolved. This image presents a great challenge to hyper-resolution incorporated with different interpolation algorithms since it has many edges and unlike textures, bad hyper-resolution results are immediately evident. Figures 4(b), 4(c), and 4(d) are hyper-resolved where bilinear, cubic spline, and probabilityfiltering-based interpolation were applied, respectively. Note that the edges in the magnified image are sharp yet lack jaggedness, especially for Figure 4(d).

The PSNRs of the hyper-resolution scheme are $23.91 \mathrm{~dB}$, $24.94 \mathrm{~dB}$, and $25.80 \mathrm{~dB}$ using bilinear, cubic spline, and probability-filtering-based interpolation, respectively. As can be seen, the hyper-resolution incorporated with the probability-filtering-based interpolation generally provides a better image quality.

The second case applied the proposed hyper-resolution scheme to a single down-sampled image shown in Figure 5(a). It can be seen that a highly down-sampled image shows serious blocking artifacts. To solve this problem, this down-sampled image was hyper-resolved using our proposed scheme where hyper-resolution was performed three times for comparison; these three hyper-resolved images are shown in Figures 5(b), 5(c), and 5(d), respectively. Obviously, blocking artifacts are further reduced, especially for the case of which interpolation with the factor $2^{3} \times 2^{3}$, shown in Figure 5(d) in which the pupils of the clown are clearly identified.

\subsection{Resolution enhancement of color images}

The results presented here show good performance, although printed here in gray-levels, using the simple approach to applying the hyper-resolution scheme to the red, green, and blue planes independently and then combining the results into a single color image.

Figure 6 illustrates the performance comparison of hyper-resolution with the bilinear, cubic spline, and probability-filtering-based interpolation methods for a color image. In the bilinear or cubic spline case, Figures 6(b) and 6(c), the aliasing effects are much more apparent and the edges are blurred where the jagged lines are quite pronounced.

The proposed scheme eliminates this problem as shown in Figure 6(d), and performs quite well as it preserves quite lots of image details with a higher contrast and sharper edges than the other two hyper-resolved images.

The PSNRs of the hyper-resolution results for Figure 6 show that relatively different improvements were obtained with the red, green, and blue colors for hyper-resolution with different interpolators. The proposed hyper-resolution 


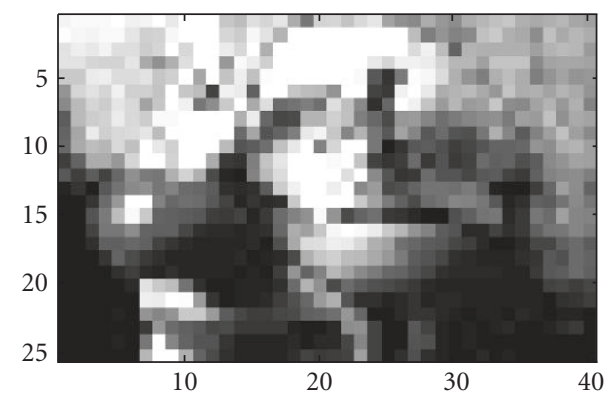

(a)

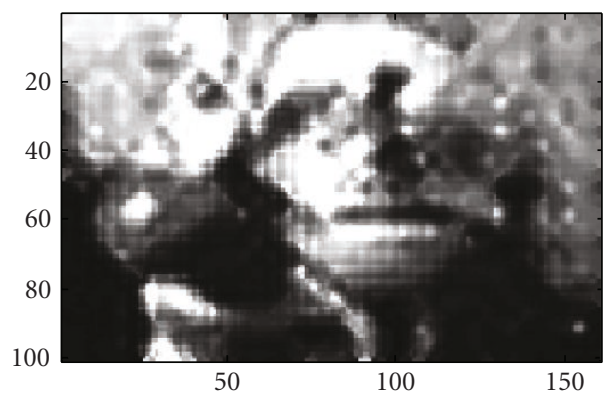

(c)

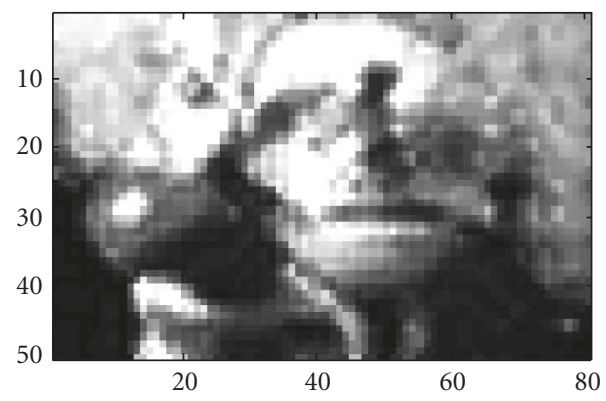

(b)

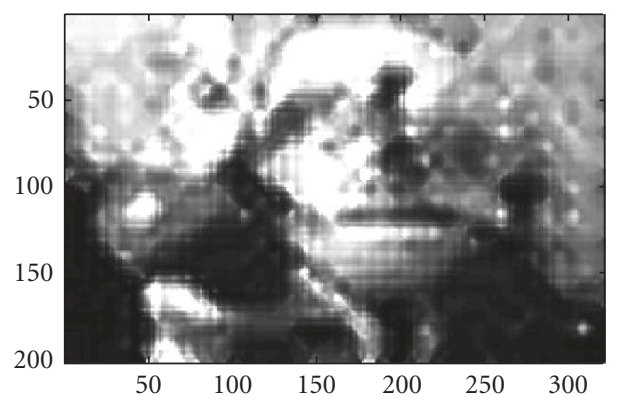

(d)

FIGURE 5: Demonstration of hyper-resolution for a single down-sampled gray-level image. (a) Down-sampled image, (b) hyper-resolved image with a factor $2 \times 2$, (c) hyper-resolved image with a factor $2^{2} \times 2^{2}$, and (d) hyper-resolved image with a factor $2^{3} \times 2^{3}$.

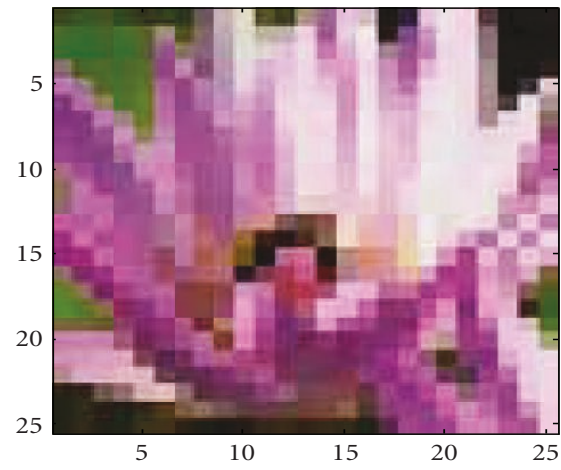

(a)

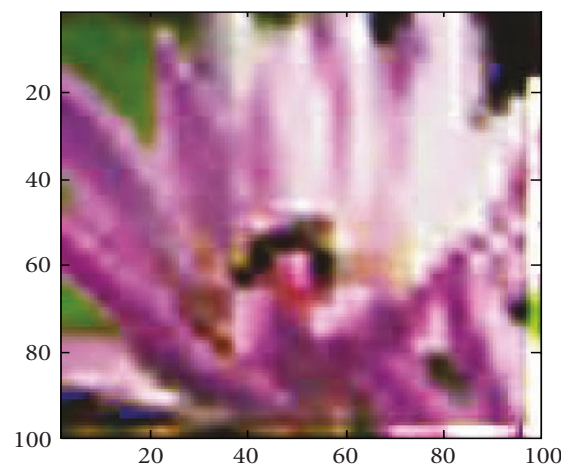

(c)

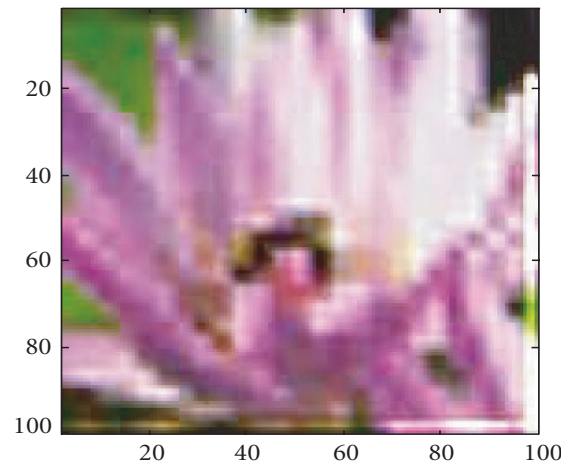

(b)

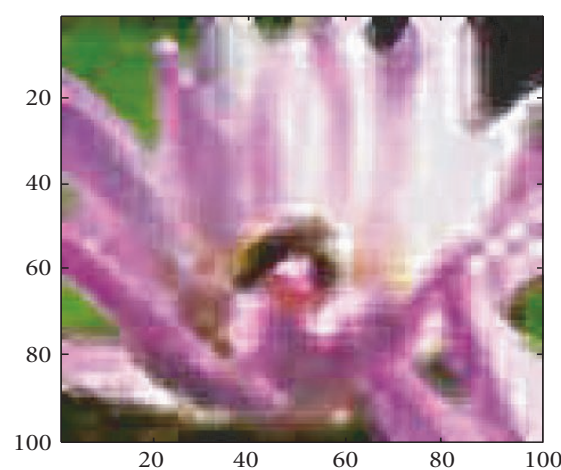

(d)

FIGURE 6: Demonstration of hyper-resolution for a single down-sampled color image. (a) Down-sampled image, (b) hyper-resolved image incorporated with bilinear interpolation, (c) hyper-resolved image incorporated with cubic spline interpolation, and (d) hyper-resolved image incorporated with probability-filtering-based interpolation. 


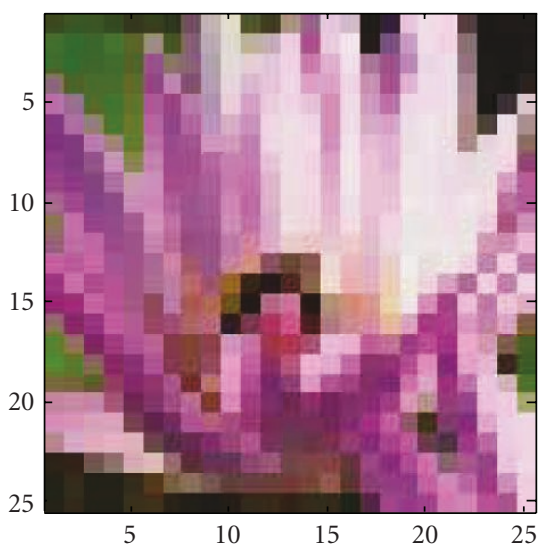

(a)

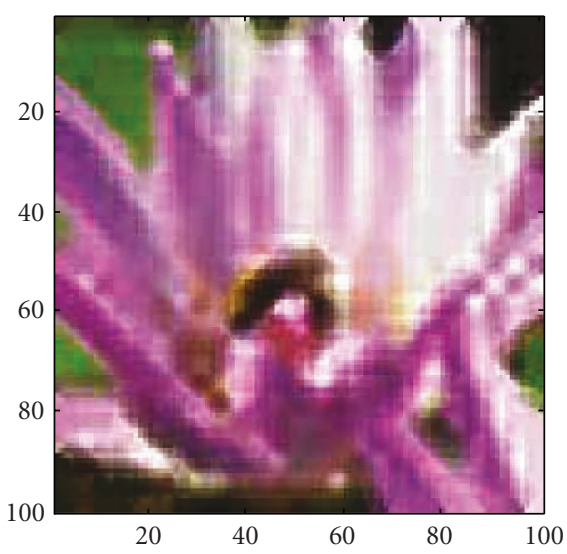

(c)

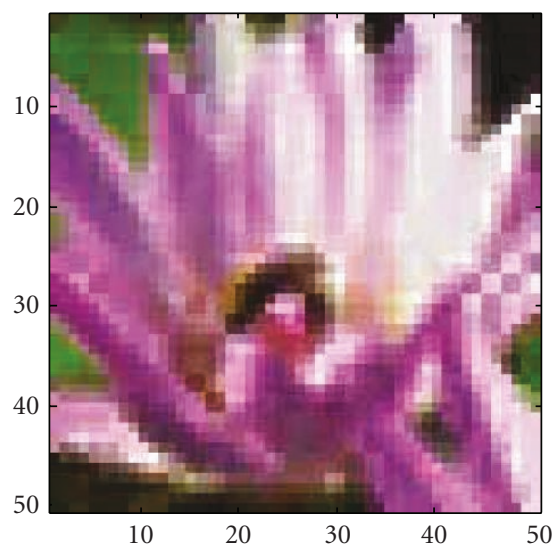

(b)

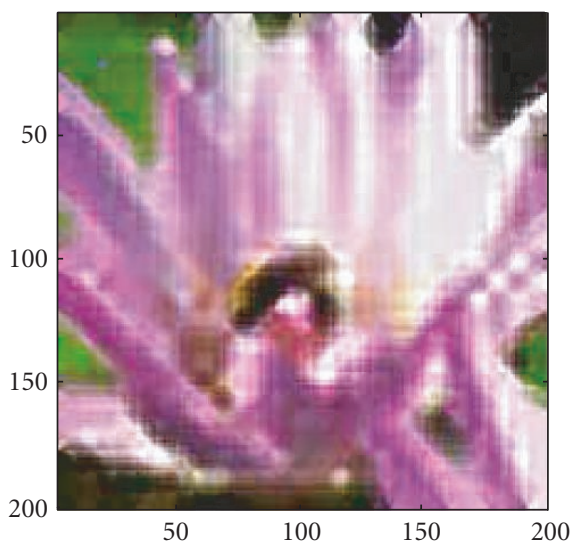

(d)

Figure 7: Demonstration of hyper-resolution for a single down-sampled color image. (a) Down-sampled image, (b) hyper-resolved image with a factor $2 \times 2$, (c) hyper-resolved image with a factor $2^{2} \times 2^{2}$, and (d) hyper-resolved image with a factor $2^{3} \times 2^{3}$.

provides generally better image quality, the PSNR of which being equal to $19.28 \mathrm{~dB}$ and outperforming $10.89 \mathrm{~dB}$ and $8.37 \mathrm{~dB}$ over the hyper-resolution using bilinear and cubic spline interpolation, respectively.

As the case of Figure 7, this down-sampled color image with serious blocking artifacts, Figure 7(a), was hyperresolved three times using our proposed method; thus, three hyper-resolved images are shown in Figures 7(b), 7(c), and 7(d), respectively. Apparently, the proposed algorithm generates hyper-resolved images with a higher visual quality, especially for the case of $2^{3} \times 2^{3}$ hyper-resolution shown in Figure 7(d).

\subsection{Resolution enhancement of noisy images}

Figure 8 shows an example of the image of a clown with Gaussian white and impulse noise. Figure 8(a) is the noisy image with the size of $50 \times 80$ pixels originally corrupted by Gaussian white noise at a PSNR equal to $16 \mathrm{~dB}$ and impulse noise at $2.5 \%$ density level; Figures $8(\mathrm{~b})$ and $8(\mathrm{c})$ are the hyper-resolved images where bilinear and cubic spline interpolators were used, respectively. It is easy to see that the noise effect of such hyper-resolved images still exists, especially for the region around the eyes. Figure 8(d) illustrates the effectiveness of using the proposed hyper-resolution scheme with the probability-filtering-based interpolation. Noise effect remains in the image where bilinear or cubic spline interpolation was used, but is reduced by using the proposed method.

\section{CONCLUSIONS}

In this paper, we have developed a hyper-resolution algorithm for enhancing image resolutions based on the hybrid scheme of the probability-filtering-based interpolation and the improved Poisson MAP super-resolution algorithm.

This paper has studied three important applications of the proposed hyper-resolution algorithm: resolution enhancement on gray-level, color, and noisy images. In these applications, the proposed hyper-resolution scheme demonstrates significant improvements over that using conventional interpolation for comparison on visual quality of the hyper-resolved images. Especially, it is more preferable of using our proposed hyper-resolution scheme than the scheme with the bilinear or cubic spline interpolator for the quality improvement of noisy images due to the probability-filtering-based interpolation with simultaneous 


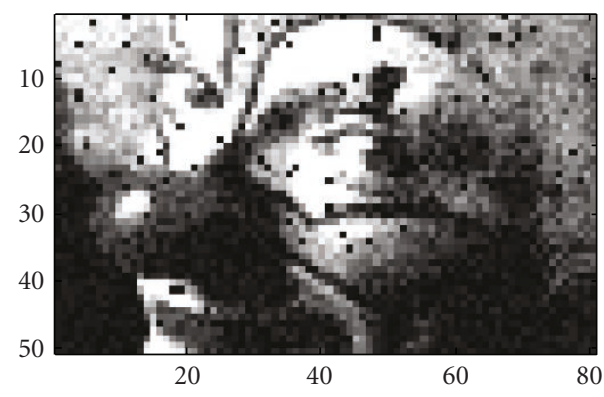

(a)

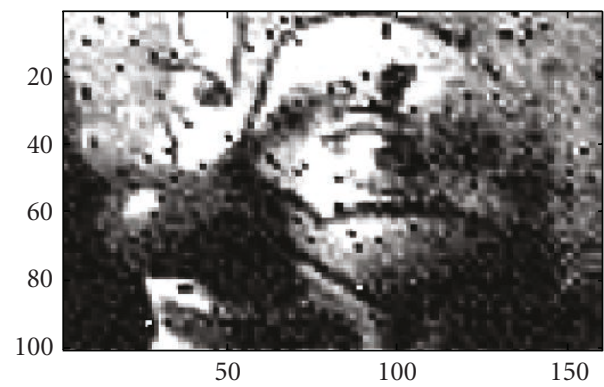

(c)

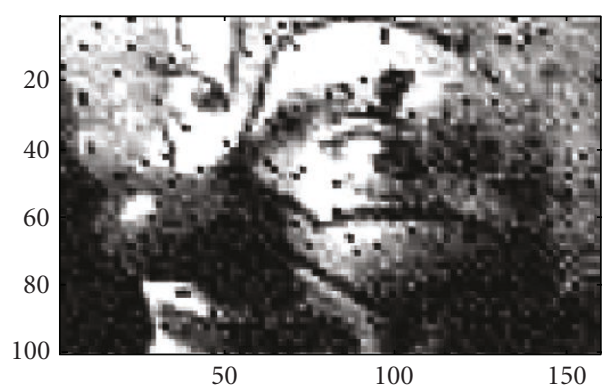

(b)

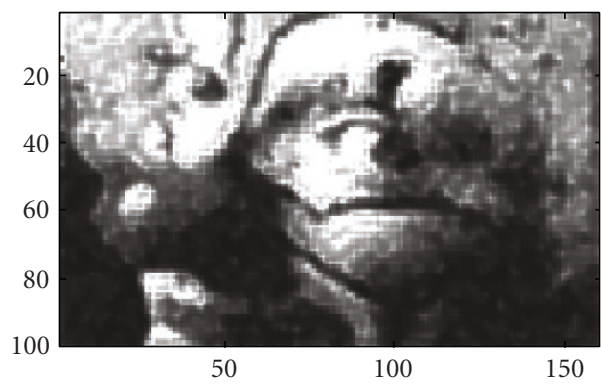

(d)

FIGURE 8: Demonstration of hyper-resolution for a single down-sampled noisy gray-level image. (a) Down-sampled image, (b) hyperresolved image incorporated with bilinear interpolation, (c) hyper-resolved image incorporated with cubic spline interpolation, and (d) hyper-resolved image incorporated with probability-filtering-based interpolation.

image interpolation and smoothing. It is anticipated that such scheme can also provide very promising reconstruction quality in demosaicing problems. Although intercorrelations among the red, green, blue colors have not been exploited in this paper, a hyper-resolution utilizing such intercorrelations would significantly improve the performance. Therefore, future work in this area will further extend the adaptive capability by incorporating image characteristics.

\section{ACKNOWLEDGMENT}

This research is substantially supported by a grant from the National Science Council in Taiwan (Project no. NSC 922213-E-236-004).

\section{REFERENCES}

[1] R. H. Chan, T. F. Chan, L. Shen, and Z. Shen, "Wavelet algorithms for high-resolution image reconstruction," SIAM Journal on Scientific Computing, vol. 24, no. 4, pp. 1408-1432, 2003.

[2] R. R. Schultz and R. L. Stevenson, "Extraction of highresolution frames from video sequences," IEEE Transactions on Image Processing, vol. 5, no. 6, pp. 996-1011, 1996.

[3] M. Elad and R. Feuer, "Super-resolution reconstruction of image sequences," IEEE Transactions on Pattern Analysis and Machine Intelligence, vol. 21, no. 9, pp. 817-834, 1999.

[4] R. C. Hardie, K. J. Barnard, and E. E. Armstrong, "Joint MAP registration and high-resolution image estimation using a sequence of undersampled images," IEEE Transactions on Image Processing, vol. 6, no. 12, pp. 1621-1633, 1997.
[5] C. Liu, H.-Y. Shum, and C.-S. Zhang, "A two-step approach to hallucinating faces: global parametric model and local nonparametric model," in Proceedings of IEEE Computer Society Conference on Computer Vision and Pattern Recognition (CVPR '01), vol. 1, pp. I-192-I-198, Kauai, Hawaii, USA, December 2001.

[6] S. Baker and T. Kanade, "Limits on super-resolution and how to break them," in Proceedings of IEEE Computer Society Conference on Computer Vision and Pattern Recognition (CVPR '00), vol. 2, pp. 372-379, Hilton Head Island, SC, USA, June 2000.

[7] H. Hou and H. Andrews, "Cubic splines for image interpolation and digital filtering," IEEE Transactions on Acoustics, Speech, and Signal Processing, vol. 26, no. 6, pp. 508-517, 1978.

[8] A. K. Jain, Fundamentals of Digital image Processing, PrenticeHall, Englewood Cliffs, NJ, USA, 1989.

[9] R. Keys, "Cubic convolution interpolation for digital image processing," IEEE Transactions on Acoustics, Speech, and Signal Processing, vol. 29, no. 6, pp. 1153-1160, 1981.

[10] K. P. Hong, J. K. Paik, H. J. Kim, and C. H. Lee, "An edge-preserving image interpolation system for a digital camcorder," IEEE Transactions on Consumer Electronics, vol. 42, no. 3, pp. 279-284, 1996.

[11] G. C. Gurski, M. T. Orchard, and A. W. Hull, "Optimal linear filters for pyramidal decomposition," in Proceedings of IEEE International Conference on Acoustics, Speech, and Signal Processing (ICASSP '92), vol. 4, pp. 633-636, San Francisco, Calif, USA, March 1992.

[12] T. Ramstad, Y. Wang, and S. K. Mitra, "Efficient image interpolation scheme using hybrid IIR Nyquist filters," Optical Engineering, vol. 31, no. 6, pp. 1277-1283, 1992. 
[13] S. Carrato, G. Ramponi, and S. Marsi, "A simple edge-sensitive image interpolation filter," in Proceedings of International Conference on Image Processing (ICIP' 96), vol. 3, pp. 711-714, Lausanne, Switzerland, September 1996.

[14] X. Li and M. T. Orchard, "New edge directed interpolation," in Proceedings of International Conference on Image Processing (ICIP '00), vol. 2, pp. 311-314, Vancouver, British Columbia, Canada, September 2000.

[15] B. Ayazifar and J. S. Lim, "PEL-adaptive model-based interpolation of spatially subsampled images," in Proceedings of IEEE International Conference on Acoustics, Speech, and Signal Processing (ICASSP' '92), vol. 3, pp. 181-184, San Francisco, Calif, USA, March 1992.

[16] B. R. Hunt and P. J. Sementilli, "Description of a Poisson imagery super-resolution algorithm," in Astronomical Data Analysis Software and System I, D. M. Worrall, C. Biemserfer, and J. Barnes, Eds., vol. 25, pp. 196-199, Astronomical Society of the Pacific, San Francisco, Calif, USA, 1992.

[17] P. J. Sementilli, M. S. Nadar, and B. R. Hunt, "Poisson MAP super-resolution estimator with smoothness constraint," in Neural and Stochastic Methods in Image and Signal Processing II, vol. 2032 of Proceedings of SPIE, pp. 2-13, San Diego, Calif, USA, July 1993.

[18] L. B. Lucy, "An iterative technique for the rectification of observed distributions," The Astronomical Journal, vol. 79, no. 6, pp. 745-765, 1974.

[19] W. H. Richardson, "Bayesian-based iterative method of image restoration," Journal of the Optical Society of America, vol. 62, no. 1, pp. 55-59, 1972.

[20] B. R. Frieden, "Restoring with maximum likelihood and maximum entropy," Journal of the Optical Society of America, vol. 62 , no. 4, pp. 511-518, 1972.

[21] M. I. Sezan and A. M. Tekalp, "Iterative image restoration with ringing suppression using themethod of POCS," in Proceedings of IEEE International Conference on Acoustics, Speech, and Signal Processing (ICASSP '88), vol. 2, pp. 1300-1303, New York, NY, USA, April 1988.

[22] M.-C. Pan and A. H. Lettington, "Smoothing images by a probability filter," in Proceedings of IEEE International Joint Symposia on Intelligence and Systems (IJSIS '98), pp. 343-346, Rockville, Md, USA, May 1998.

[23] M.-C. Pan, "A novel blind super-resolution technique based on the improved Poisson maximum a posteriori algorithm," International Journal of Imaging Systems and Technology, vol. 12, no. 6, pp. 239-246, 2002.

[24] M. Unser, A. Aldroubi, and M. Eden, "B-spline signal processing. I. Theory," IEEE Transactions on Signal Processing, vol. 41, no. 2, pp. 821-833, 1993.

[25] C. Lee, M. Eden, and M. Unser, "High-quality image resizing using oblique projection operators," IEEE Transactions on Image Processing, vol. 7, no. 5, pp. 679-692, 1998.

[26] M. Unser, "Splines: a perfect fit for signal and image processing," IEEE Signal Processing Magazine, vol. 16, no. 6, pp. 22-38, 1999.

[27] R. Kimmel, "Demosaicing: image reconstruction from color CCD samples," IEEE Transactions on Image Processing, vol. 8, no. 9, pp. 1221-1228, 1999.
Min-Cheng Pan was born in Kaohsiung, Taiwan, on September 19, 1966. He received the B.Eng. and M.S. degrees from National Chiao Tung University and National Taiwan University in 1988 and 1990, respectively, and the Ph.D. degree in the Applied Optics Group of Department of Physics from The University of Reading, England, in 1999. He is currently an Associate Professor in the Department of Computer Science and Information Engineering, Tung-Nan Institute of Technology, and a Research Consultant in industry since August 1999. His research interests include image super-resolution, image reconstruction, numerical analysis, finite element method, surveillance systems, and medical imaging systems.

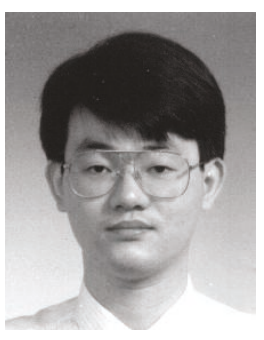

